Article

\title{
Hybrid Phosphate-Alumina Iron-Based Core-Shell Soft Magnetic Composites Fabricated by Sol-Gel Method and Ball Milling Method
}

\author{
Yifan Pan ${ }^{1}$, Liwei Qian ${ }^{1}$, Xiang Wang ${ }^{2}$, Jingguang Peng ${ }^{2, *}$ and Wei Lu ${ }^{1, *}$ \\ 1 Shanghai Key Lab of D\&A for Metal-Functional Materials, School of Material Science and Engineering, \\ Tongji University, 201800 Shanghai, China; panyifan1017@163.com (Y.P.); qianlw_tj@163.com (L.Q.) \\ 2 Shanghai Automotive Powder Metallurgy Co., 201900 Shanghai, China; xiangw@shautopm.com.cn \\ * Correspondence: jingguangp@shautopm.com.cn (J.P.); weilu@tongji.edu.cn (W.L.); \\ Tel.: +86-21-6958-1508 (W.L.)
}

Received: 9 January 2020; Accepted: 10 February 2020; Published: 17 February 2020

\begin{abstract}
Novel Fe-based soft magnetic composites (SMCs) with hybrid phosphate-alumina layers were prepared by both sol-gel and ball-milling methods. The effects of the fabrication methods and the addition of $\mathrm{Al}_{2} \mathrm{O}_{3}$ particles on the microstructure and the soft magnetic performance of SMCs were studied. The formation of the hybrid phosphate- $\mathrm{Al}_{2} \mathrm{O}_{3}$ shell not only leads to the decrease of the total core loss, but also results in the reduction of the permeability and saturation magnetization. However, the degree of decrease caused by the different methods were not identical. The sample with $8 \% \mathrm{Al}_{2} \mathrm{O}_{3}$ prepared by the sol-gel method showed the best magnetic performance, exhibiting a high-amplitude permeability $\left(\mu_{\mathrm{a}}\right)$ of 85.14 and a low total core loss $(P \mathrm{~s})$ of $202.3 \mathrm{~W} / \mathrm{kg}$ at $50 \mathrm{mT}$ and $100 \mathrm{kHz}$. The hysteresis loss factor and the eddy current loss factor were obtained by loss separation. The results showed that the samples with the same $\mathrm{Al}_{2} \mathrm{O}_{3}$ content prepared by different methods exhibited almost the same total core loss. However, the contribution of the hysteresis loss and the eddy current loss showed an obvious difference in behavior because of the change of the particle shapes and the refinement of the particle size during the ball-milling process.
\end{abstract}

Keywords: soft magnetic composites; sol-gel method; ball-milling method; loss separation

\section{Introduction}

The eddy currents which appear in ferromagnetic materials are caused by the magnetization in an alternating current (AC) magnetic field as a consequence of Faraday's law. The ferromagnetic materials that were magnetized in an AC magnetic field would internally-generate the eddy current, a long-existing barrier that limited the applications of the devices at medium to high frequency because of the increase in eddy current loss and the reduction of the permeability [1,2].

Owing to their superior properties, including high permeability, high-frequency stability, 3D isotropy magnetic field, high productivity and, especially, the high electrical resistivity, iron-based soft magnetic composites (SMCs) with a special core-shell structure [3] have attracted broad academic interest and investigations in order to satisfy the requirements of applications in power electronics, transformers and electric motors at medium to high frequency $[4,5]$. The iron-based SMCs are conventionally prepared by coating insulating materials onto the pure iron matrix to block the path of closed-current loops and the compaction and annealing process [6]. Therefore, the selection of appropriate insulating materials with high electric resistivity and good adhesive strength is vital to minimize the eddy current loss of the SMC cores [7].

The coating layers can be divided into two principal types: organic materials and inorganic materials. Organic materials such as silicon resin [8] and epoxy [9] are the most popular insulating 
compounds due to their high electric resistivity and good flexibility. However, the application of organic materials in insulating layers is restricted due to their low thermal stability, which may not permit high-temperature heat treatment to eliminate the internal stress that is introduced during the preparation process. Thus, inorganic materials, including phosphates [10], oxides such as $\mathrm{SiO}_{2}$, $\mathrm{Al}_{2} \mathrm{O}_{3}, \mathrm{ZrO}_{2}, \mathrm{Fe}_{3} \mathrm{O}_{4}$ [11-13] and ferrites such as Ni-Zn ferrites and Mn-Zn ferrites [14,15], have gained greater attention and are increasingly considered as promising insulating materials because of their superior electrical resistance and temperature resistance. Peng et al. [15] fabricated Mn-Zn ferrite-coated iron-based composite particles by a co-precipitation method and studied the soft magnetic properties of the composites. Wu et al. [16] prepared $\mathrm{Fe} / \mathrm{SiO}_{2}$ composite particles via a reverse micro-emulsion method and investigated the effects of the addition amounts of $\mathrm{SiO}_{2}$ and the heat-treatment temperatures on the permeability and the total core loss of the composites. Though the above composite particles $[15,16]$ have been fabricated, it was difficult to ensure the homogeneity of the coatings to be applied in industrialization. In addition, the insulating layer is easily peeled off because of the poor adhesiveness to the matrix, which would result in the direct contact between core particles. In comparison to the oxides and nano-ferrites, phosphates show the advantage of better adhesiveness to the iron matrix, good homogeneity of coatings and the potential of industrialization because of the simple preparation process and more limited equipment required. However, a previous paper [17] has reported that the phosphate coatings would crystalize or decompose at a relatively high temperature (above $500^{\circ} \mathrm{C}$ ), dramatically decreasing the electric resistivity. Huang et al. [18] investigated the effect of annealing temperatures on the composition and the magnetic properties of the phosphate-coated SMCs; their results showed that phosphate coatings degrade into iron phosphide after annealing at $600{ }^{\circ} \mathrm{C}$, and the magnetic properties worsened as a result. Owing to the high bond energy of the Al-O covalent bond, $\mathrm{Al}_{2} \mathrm{O}_{3}$ possess high electrical resistivity and high temperature resistance. However, neither the single phosphate coating nor the alumina could satisfy the requirement of the application at high frequency. Therefore, hybrid phosphate-alumina iron-based soft magnetic composites are promising to effectively reduce the total core loss to broaden the frequency of industrial applications.

Owing to the advantages such as low equipment requirements, easy operation and economic efficiency, the mechanical milling method and the sol-gel method are two of the most widely used methods to prepare SMCs. In this work, novel hybrid phosphate-alumina iron-based SMCs were fabricated first by phosphating and then by coating with $\mathrm{Al}_{2} \mathrm{O}_{3}$ via two methods, namely sol-gel and mechanical milling. The hybrid-coated SMCs retained relatively high permeability and showed very low core loss. The effects of the $\mathrm{Al}_{2} \mathrm{O}_{3}$ content and the different preparation methods on the soft magnetic properties of the composites were studied in detail.

\section{Preparation and Characterization}

Gas-atomized iron powders with a particle size ranging from $75 \mu \mathrm{m}$ to $250 \mu \mathrm{m}$ were conducted to achieve the desired size ratio, annealed at $700{ }^{\circ} \mathrm{C}$ for some time, twice cleaned in absolute ethyl alcohol, and dried at $60^{\circ} \mathrm{C}$ for five hours before the passivation. Then, $50 \mathrm{~g}$ iron powders were mixed with a certain amount of ethanol-diluted orthophosphate acid with a concentration of $0.01 \mathrm{~g} / \mathrm{mL}$, and the mixture were stirred in a solution of $65^{\circ} \mathrm{C}$ until the solvent completely evaporated to obtain the phosphate-coated particles.

The sol-gel method and the ball-milling method were used to prepare Fe @ phosphate particles to obtain powders with the hybrid coating. A certain amount of aluminum oxides were dissolved in $100 \mathrm{~mL}$ of ethanol and was magnetically stirred at room temperature for $1 \mathrm{~h}$ to obtain the aluminum sol. Then, some as-prepared $\mathrm{Fe} /$ phosphate powders were dispersed in the solution using a spiral mixer for $15 \mathrm{~min}$. A mixture of distilled water and ethanol (1:3(V/V)) was added dropwise, acetic acid was added to adjust the $\mathrm{pH}$ value to around four, and the mixture was continuously stirred for $1.5 \mathrm{~h}$. Subsequently, the suspension was separated and washed with ethanol three times and dried. Finally, the powders were calcinated at $600^{\circ} \mathrm{C}$ for $2 \mathrm{~h}$ under a nitrogen atmosphere. 
As for the ball-milling method, the weight ratio of ball to powders was set to 10:1 and a mixture of the as-prepared $\mathrm{Fe} /$ phosphate powders and the $\alpha-\mathrm{Al}_{2} \mathrm{O}_{3}$ nanoparticles with the weight percent of $2 \mathrm{wt} \%-8 \mathrm{wt} \%$ were carried out at a speed of $200 \mathrm{rpm}$ for $30 \mathrm{~min}$. All of the above powders were mixed with the lubricant of Zinc stearate $(0.5 \mathrm{wt} \%)$ and were pressed at an uniaxial pressure of $800 \mathrm{MPa}$ at room temperature to obtain the ring-shaped compacts with an external diameter of $20 \mathrm{~mm}$, an inside diameter of $12 \mathrm{~mm}$ and height of approximately $3.4 \mathrm{~mm}$.

An X-ray diffractometer using $\mathrm{Cu}-\mathrm{K} \alpha$ radiation was used to analyze the phases of the samples. The morphology and composition of the powders were detected by a scanning electron microscopy (SEM, Nova NanoSEM 450, FEI, USA) equipped with an energy-dispersive X-ray Spectroscope (EDS, Ultra, EDAX, USA), whose resolutions were $1.0 \mathrm{~nm}$ at $15 \mathrm{kV}$ and $0.8 \mathrm{~nm}$ at $30 \mathrm{kV}$. All of the SEM images were magnified 1000 times and a metallographic analyzer produced by a Shanghai optical instruments factory was used to characterize the microstructure. The soft magnetic properties of the ring-shaped compacts were determined by a hysteresis looper (MATS 2010SA) at a frequency ranging from $5 \mathrm{kHz}$ to $100 \mathrm{kHz}$ and a magnetic excitation level of $0.05 \mathrm{~T}$; the uncertainty of measured $\mu_{\mathrm{a}}$ and total loss were less than 3\% and 5\%, respectively. The vibrating sample magnetometer (VSM) was conducted to measure the saturation magnetization of the compacts; the accuracy and repeatability were less than $1 \%$ and $5 \%$, respectively.

\section{Results and Discussion}

\subsection{SMCs with Single Phosphate Coating}

Figure 1 shows the SEM images of the raw iron powders, the phosphated powders, and the EDS (energy disperse spectrum) analysis and maps of the phosphated powders. Results suggest that the coated powders keep the same irregular shape and only show a rougher surface than the raw iron powders. Figure 1c, which displays the EDS analysis of the selected area of the phosphated iron particle, indicates the existence of $\mathrm{Fe}, \mathrm{O}$ and $\mathrm{P}$ on the surface of the phosphated particles. In addition, the EDS maps show that the iron, oxygen and phosphate elements distribute on the surface of the coated powders. Therefore, it can be concluded that the phosphate insulating layers were formed during the phosphating process.
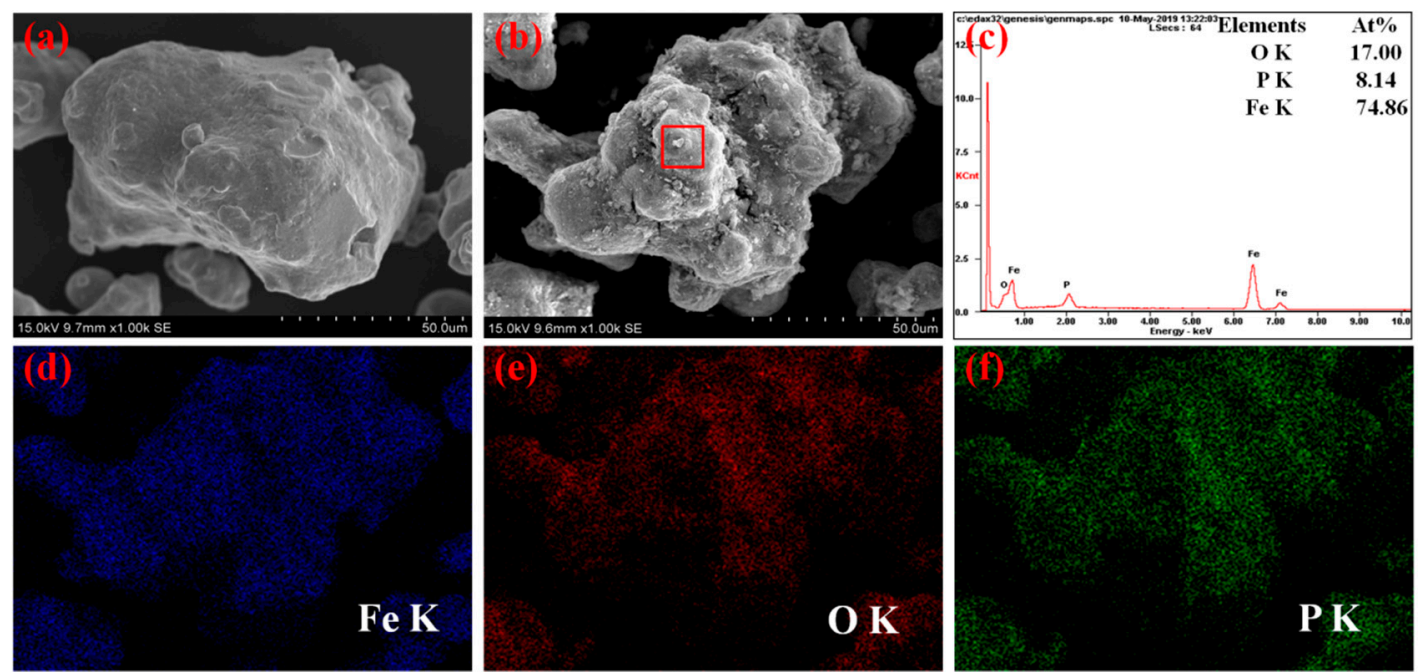

Figure 1. SEM images of the powders (a) pure iron powder, (b) phosphated iron powder, (c) EDS analysis of the selected area and EDS maps of (d) iron, (e) oxygen and (f) phosphorus of phosphated powders. 
Figure 2 reveals the influence of the phosphating on the permeability and the total core loss of the compacts at $50 \mathrm{mT}$ external magnetic field. The permeability of the as-coated sample is $127.7 \mathrm{at} 5 \mathrm{kHz}$ and is not as high as that of the pure iron sample due to the magnetic dilution effect of the non-magnetic materials [19]. The presence of non-magnetic phosphate between the magnetic cores plays the same role as air gaps, which would create a demagnetizing field in the entire magnetic circuit, resulting in lower permeability at low frequency. However, owing to the greater frequency stability, the permeability of the coated sample is superior to that of the pure iron sample at $100 \mathrm{kHz}$. At high frequency, the phosphate with strong resistivity leads to a lower eddy current. The increased eddy current caused by the high-frequency AC field in the pure iron sample circulates in the opposite direction to the applied field, which results in the decrease of the excited magnetic field and permeability [17,20].
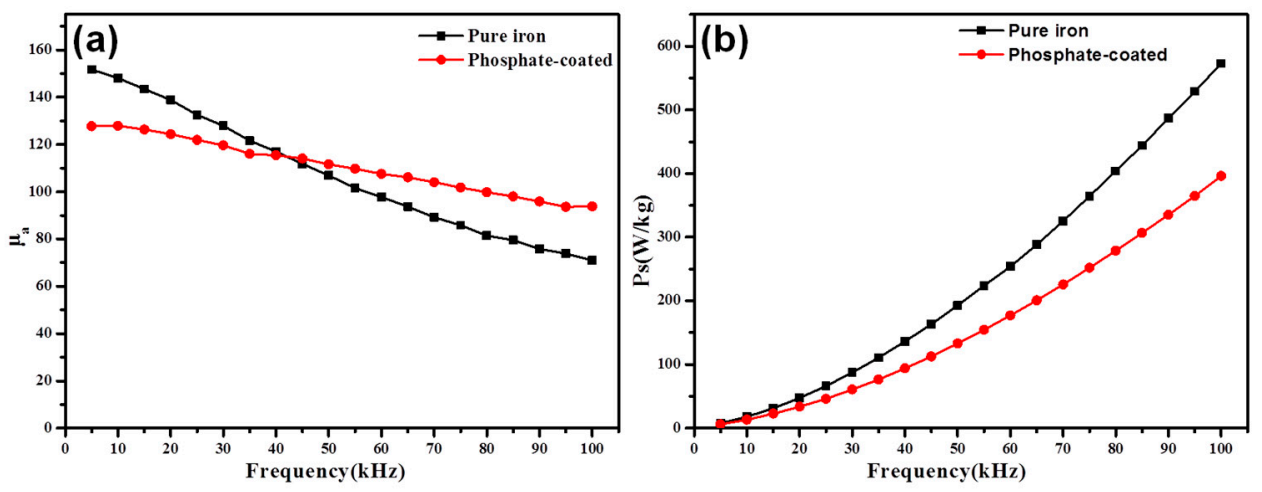

Figure 2. The effects of the phosphating process on the soft magnetic properties of the samples (a) the amplitude permeability and (b) the core loss.

The total core loss of the as-coated sample (shown in Figure 2b) is approximately two thirds of the pure iron sample at $100 \mathrm{kHz}$. This can be explained by the blocking effect of the phosphate insulation layer on the inter-particle eddy current loss, which is the main contributor to the total core loss at high frequency.

\subsection{SMCs with Hybrid Phosphate-Alumina Coating}

The XRD patterns of the pure iron particles and the hybrid-coated particles are displayed in Figure 3. The diffraction peaks at $2 \theta=44.667^{\circ}, 65.000^{\circ}$ and $82.366^{\circ}$ correspond to the (110), (200) and (211) planes of the $\alpha$-Fe phase (JCPDS 06-0696). The XRD patterns of the coated powders are not obviously different from those of the original. Only the diffraction peak intensity of the $\alpha$-Fe phases of the hybrid-coated powders via the ball-milling method decreases slightly and no other diffraction peaks could be observed. This could be explained primarily by the possibility that the content of the phosphate layer is too small to be detected, or that the phosphate layer is amorphous [18]. Previous research has reported that amorphous $\mathrm{Al}_{2} \mathrm{O}_{3}$ would transfer to $\delta-\mathrm{Al}_{2} \mathrm{O}_{3}, \theta-\mathrm{Al}_{2} \mathrm{O}_{3}$ and $\alpha-\mathrm{Al}_{2} \mathrm{O}_{3}$ at $750{ }^{\circ} \mathrm{C}, 1000{ }^{\circ} \mathrm{C}, 1100{ }^{\circ} \mathrm{C}$, respectively [21]. Therefore, preparing the $\mathrm{Al}_{2} \mathrm{O}_{3}$ particles by the sol-gel method and calcining at $600{ }^{\circ} \mathrm{C}$ could only obtain amorphous $\mathrm{Al}_{2} \mathrm{O}_{3}$. As for the ball-milling method, the broadening of the $\alpha$-Fe peak may be due to the small crystallites and their lattice distortions [22]. The grain refinement and the plastic deformation during the ball-milling process would also decrease the intensity of the diffraction peaks. 


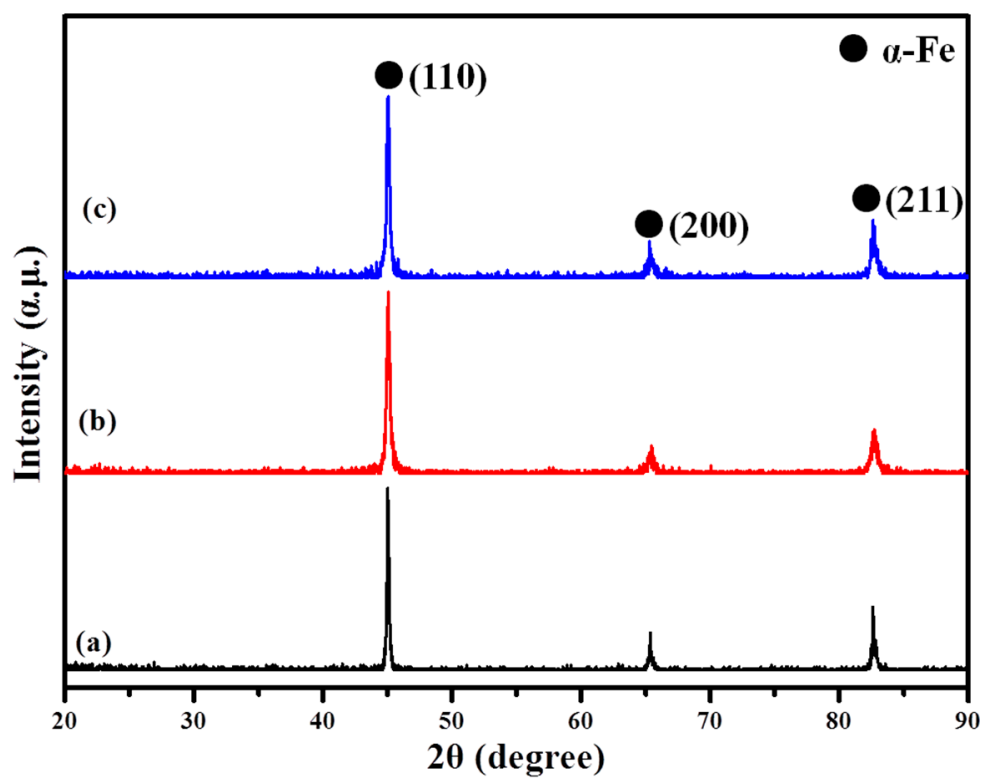

Figure 3. The XRD patterns of the powders (a) pure iron; (b) hybrid coating via the ball-milling method with $8 \% \mathrm{Al}_{2} \mathrm{O}_{3} ;$ (c) hybrid coating via the sol-gel method with $8 \% \mathrm{Al}_{2} \mathrm{O}_{3}$.

SEM images and EDS maps of the hybrid-coated powders via ball milling are exhibited in Figure 4. A change of the particle shape from the irregular shape to a flake shape after the ball-milling process could be observed in Figures 4a and 2a. Traces of aluminum, oxygen and phosphorus are uniformly detected on the particle surface in the EDS maps (Figure $4 \mathrm{~b}-\mathrm{d}$ ), suggesting the formation of the core-shell structured Fe @ phosphate @ $\mathrm{Al}_{2} \mathrm{O}_{3}$ SMCs.
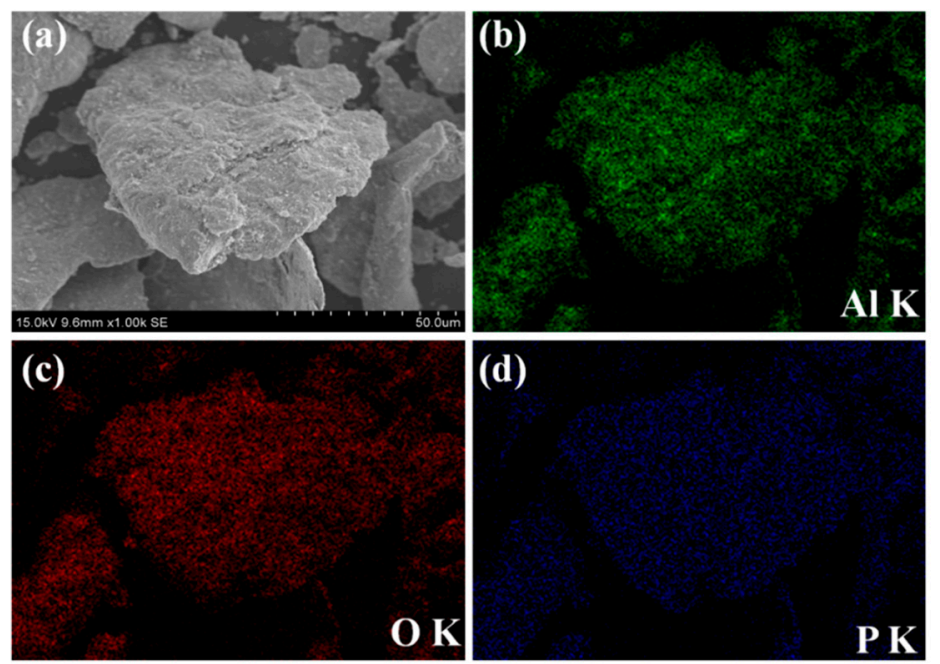

Figure 4. SEM images of (a) hybrid-coated powders with $8 \% \mathrm{Al}_{2} \mathrm{O}_{3}$ via ball milling and EDS maps of (b) aluminum, (c) oxygen and (d) phosphorus.

Figure 5a-d indicates that increasing the $\mathrm{Al}_{2} \mathrm{O}_{3}$ content results in thicker and more uniform insulating layers. No obvious insulating layers could be seen with the addition of $2 \% \mathrm{Al}_{2} \mathrm{O}_{3}$. Insulating layers were evident with additional amounts of $6 \% \mathrm{Al}_{2} \mathrm{O}_{3}$, but some parts of the matrix particles still contacted directly. Further increasing the additional amount of $\mathrm{Al}_{2} \mathrm{O}_{3}$ to $8 \%$ leads to complete insulating layers. However, more pores could be observed with the increasing $\mathrm{Al}_{2} \mathrm{O}_{3}$ content. 


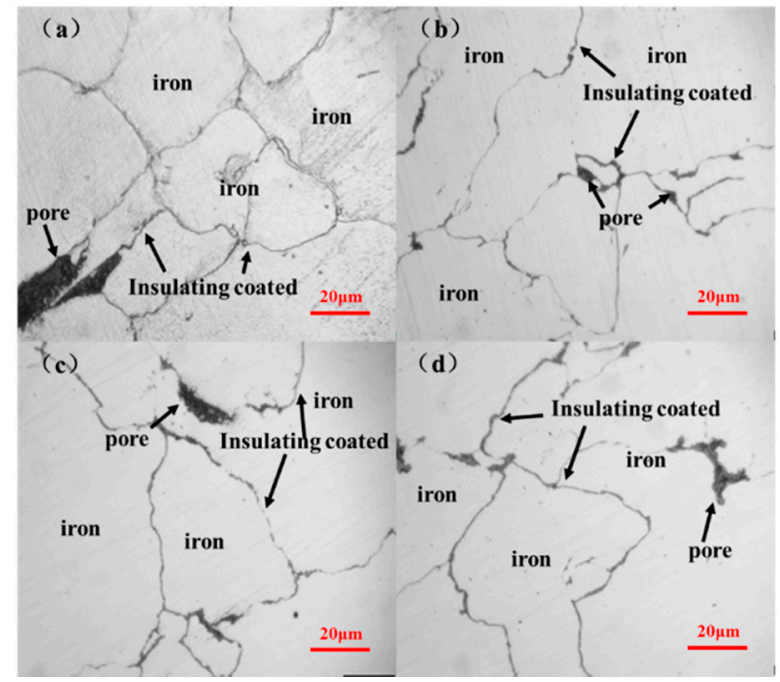

Figure 5. Metallographs of the hybrid-coated compacts by the ball-milling method with various additional amounts of $\mathrm{Al}_{2} \mathrm{O}_{3}$ (a) $2 \%$; (b) $4 \%$; (c) $6 \%$; (d) $8 \%$.

To further analyze the microstructure: the SEM (scanning electron microscopy) images of the polished surface of the $\mathrm{Fe} @$ phosphate $@ \mathrm{Al}_{2} \mathrm{O}_{3} \mathrm{SMC}$ compact were characterized. In addition, the EDS maps were shown to verify the microstructure (Figure 6). The iron matrix particles were firmly encapsulated by a thin coating with a core-shell structure via the ball-milling method. the fact that the $\mathrm{P}$ and $\mathrm{Al}$ elements only distributed around the matrix powder and the Fe element distributed in almost the whole visual area could strongly prove the nature of the composite interface microstructure.
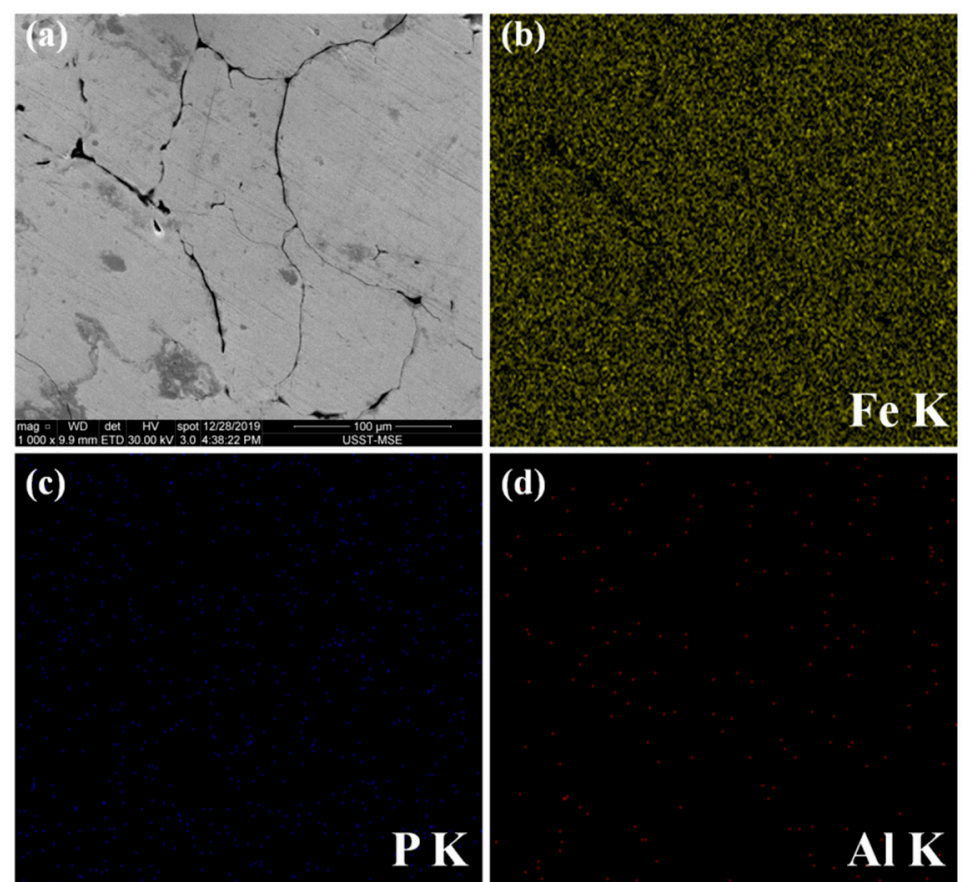

Figure 6. SEM images of (a) the polished surface of Fe @ phosphate @ $\mathrm{Al}_{2} \mathrm{O}_{3}$ SMC compact and EDS maps of (b) iron, (c) phosphorus and (d) aluminum.

Figure 7 reveals the hysteresis loops of the matrix iron particles and the hybrid-coated particles prepared by the ball-milling method with the various additional amounts of $\mathrm{Al}_{2} \mathrm{O}_{3}$. The saturated magnetization of raw pure iron is approximately $205 \mathrm{emu} / \mathrm{g}$. All the hybrid-coated powders display excellent properties of saturated magnetization of approximately $160 \mathrm{emu} / \mathrm{g}$ to $190 \mathrm{emu} / \mathrm{g}$. It is widely 
accepted that the saturated magnetization mainly depends on the composition and the purity of the materials [1], and that introducing non-magnetic phosphate and $\alpha-\mathrm{Al}_{2} \mathrm{O}_{3}$ would dilute the percentage of magnetic phases and reduce the $M s$ as a result. The decreasing trend of the saturated magnetization in Figure 7 is due to the increasing content of $\mathrm{Al}_{2} \mathrm{O}_{3}$ in insulating layers.

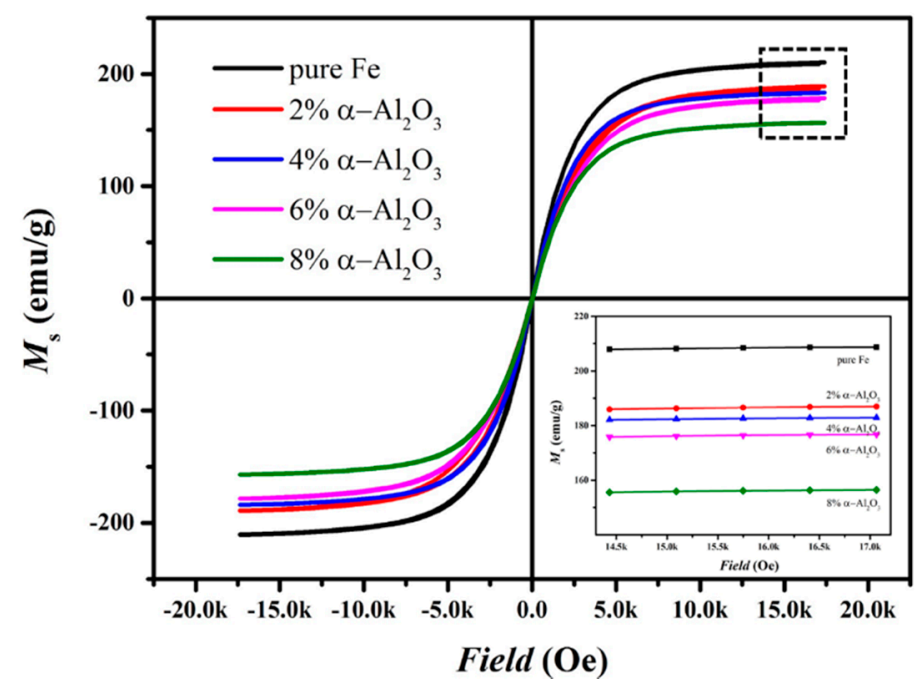

Figure 7. Magnetic hysteresis loops of the pure iron and the hybrid-coated powders prepared by the ball-milling method with the various additional amount of $\mathrm{Al}_{2} \mathrm{O}_{3}$.

Figure 8 reveals the amplitude permeability as a function of frequency of the samples prepared by the ball-milling method (Figure $8 \mathrm{a}$ ) and the sol-gel method (Figure 8b) with the same additional amount of $\mathrm{Al}_{2} \mathrm{O}_{3}$. The amplitude permeability of the hybrid-coated SMCs prepared by both methods exhibit excellent frequency stability at the measured frequency, which could be attributed to the better integrity of the insulation layers shown in Figure 5. Increasing the content of $\mathrm{Al}_{2} \mathrm{O}_{3}$ causes a tendency of permeability decrement, and the permeability of the samples with $8 \% \mathrm{Al}_{2} \mathrm{O}_{3}$ reach 77.24 (ball milling method) and 85.14 (sol-gel method), respectively, at $100 \mathrm{kHz}$, which are $\sim 82.4 \%$ and $~ 90 \%$ of that of phosphate-coated powders (93.7). The results could be explained by the following reasons. On the one hand, the permeability has a positive correlation with the $M$ s and the magnetism would be diluted by the non-magnetic $\mathrm{Al}_{2} \mathrm{O}_{3}$, as shown in Figure 6. On the other hand, a higher porosity with an increasing $\mathrm{Al}_{2} \mathrm{O}_{3}$ content could be concluded from Figure 5. The decreasing magnetic permeability is usually attributed to the inter-particle gap effect, as a source of the demagnetizing field [1]. As a result, the density of the SMCs reduces and results in the decreasing of the permeability.
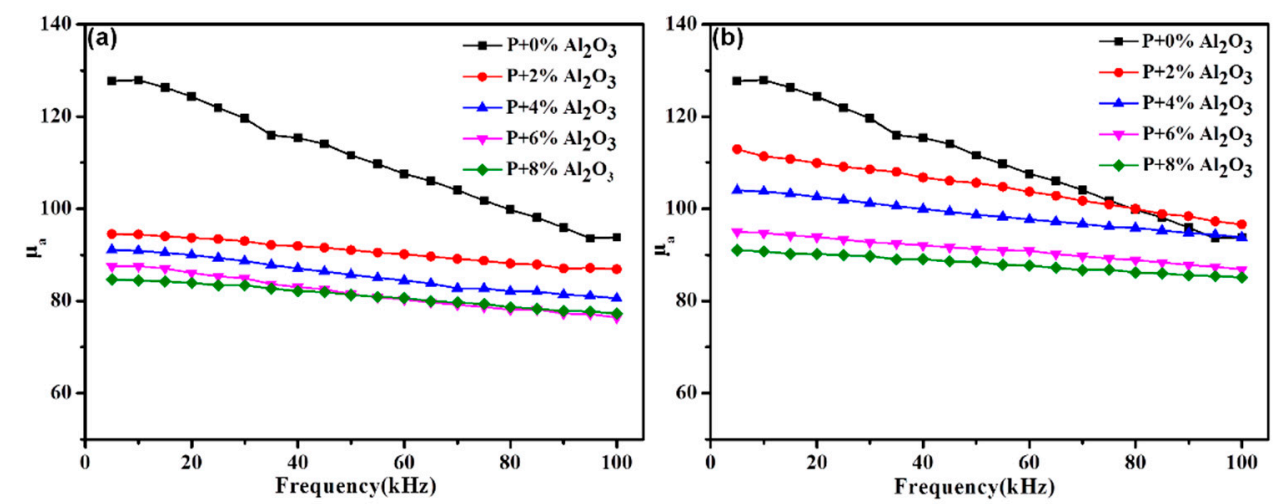

Figure 8. The amplitude permeability of the hybrid-coated samples by (a) ball milling and (b) sol-gel with the same additional amount of $\mathrm{Al}_{2} \mathrm{O}_{3}$. 
The difference of the permeability caused by the fabrication technique could mainly be attributed to the grain size. As it is accepted that the magnetization is mainly down to the movement and the rotation of the domain walls, the required energy of the domain wall movement is less than that of the domain wall rotation. As the grain size decreases, the number of domain walls decreases and the contribution of the domain wall rotation to the magnetization is greater than that of the domain wall movement [22]. The effect of the ball-milling process is to decrease the grain size; therefore, the permeability of the SMCs fabricated by this method is not as high as that prepared by the sol-gel method.

Figure 9 depicts the influence of the $\mathrm{Al}_{2} \mathrm{O}_{3}$ content on the total core loss of the SMCs samples prepared by the different methods. It can be seen that the effect of the preparation methods on the total core loss is slight. Compared with the phosphate-coated samples, the total core loss of hybrid-coated samples decreases by $\sim 49 \%$ (202.3 at $100 \mathrm{kHz}$ by the sol-gel method) and $\sim 47 \%$ (210.8 at $100 \mathrm{kHz}$ by the ball-milling method) with $8 \% \mathrm{Al}_{2} \mathrm{O}_{3}$. To further explore the effects of the $\mathrm{Al}_{2} \mathrm{O}_{3}$ content and the fabrication methods on the total core loss of SMCs samples, the total core loss curves were investigated by the loss separation method. The total core loss $\left(P_{t}\right)$ at a weak field and a relatively low frequency could be separated and written as [4,23]:

$$
P_{\mathrm{tol}}=P_{\mathrm{h}}+P_{\mathrm{e}} \approx K_{\mathrm{h}} f+K_{\mathrm{e}} f^{2}
$$

where $f$ is the frequency, the constant $K_{\mathrm{h}}$ and $K_{\mathrm{e}}$ are the hysteresis loss factor and the eddy current loss factor, respectively. $K_{\mathrm{h}}$ and $K_{\mathrm{e}}$ are obtained based on Equation (1) from the curves of the total core loss and the loss-separation fitting.
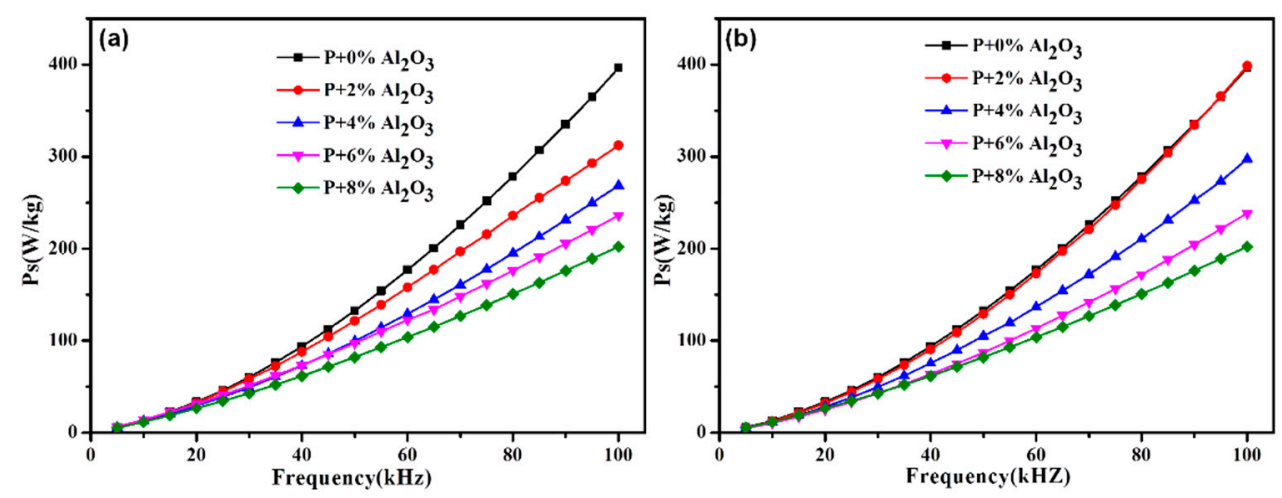

Figure 9. The total core loss of the hybrid-coated samples prepared by (a) ball milling and (b) sol-gel with the same addition amount of $\mathrm{Al}_{2} \mathrm{O}_{3}$.

Table 1 displays the $K_{\mathrm{h}}, K_{\mathrm{e}}$ and the standard error of the samples with different additional amounts of the $\mathrm{Al}_{2} \mathrm{O}_{3}$ fabricated by different methods. The $K_{\mathrm{h}}$ shows a slight increasing trend with the elevated $\mathrm{Al}_{2} \mathrm{O}_{3}$ amount, which is attributed to the higher porosity, as shown in Fig. 5 . However, the $K_{\mathrm{e}}$ exhibits an opposite, decreasing trend. The $K_{e}$ of the sample with $8 \% \mathrm{Al}_{2} \mathrm{O}_{3}$ is only one-quarter of that of the phosphate-coated sample. The $K_{\mathrm{h}}$ and $K_{\mathrm{e}}$ of samples prepared by the ball-milling method is higher and lower, respectively, than that of samples by the sol-gel method at the same $\mathrm{Al}_{2} \mathrm{O}_{3}$ content.

Table 1. $K_{\mathrm{h}}, K_{\mathrm{e}}$ and their standard error of samples with various content of $\mathrm{Al}_{2} \mathrm{O}_{3}$ by different methods.

\begin{tabular}{|c|c|c|c|c|c|c|c|c|}
\hline $\begin{array}{l}\text { Content of } \\
\mathrm{Al}_{2} \mathrm{O}_{3}(\%)\end{array}$ & $\begin{array}{c}K_{\mathrm{h}} \\
\text { (Sol-Gel) }\end{array}$ & $\begin{array}{l}\text { Standard } \\
\text { Error }\end{array}$ & $\begin{array}{l}K_{\mathrm{h}}(\text { Ball } \\
\text { Milling) }\end{array}$ & $\begin{array}{l}\text { Standard } \\
\text { Error }\end{array}$ & $\begin{array}{c}K_{\mathrm{e}} \\
\text { (Sol-Gel) }\end{array}$ & $\begin{array}{l}\text { Standard } \\
\text { Error }\left(10^{-4}\right)\end{array}$ & $\begin{array}{l}K_{\mathrm{e}}(\text { Ball } \\
\text { Milling) }\end{array}$ & $\begin{array}{l}\text { Standard } \\
\text { Error }\left(10^{-4}\right)\end{array}$ \\
\hline 0 & 1.4924 & 0.05382 & 1.4924 & 0.05382 & 0.02547 & 4.979 & 0.02547 & 4.979 \\
\hline 2 & 1.2677 & 0.03233 & 1.3307 & 0.04673 & 0.02764 & 2.990 & 0.01841 & 9.737 \\
\hline 4 & 1.3128 & 0.02956 & 1.3841 & 0.03153 & 0.01706 & 2.735 & 0.01453 & 2.917 \\
\hline 6 & 1.1807 & 0.02567 & 1.4550 & 0.02317 & 0.01248 & 2.375 & 0.00902 & $2 / 143$ \\
\hline 8 & 1.3268 & 0.02202 & 1.5068 & 0.02202 & 0.00731 & 2.037 & 0.00631 & 2.037 \\
\hline
\end{tabular}


Density, particle size and residual stress play important roles in hysteresis loss [24]. The influence of the ball-milling process on particles could be divided into two parts: particle size and particle shape. Figures 2a and 4a suggest that the morphology of the particles transforms from irregular shape to flake shape after the ball-milling process, which deteriorates the compressibility of the powders and results in the lower density and higher porosity. In addition, the smaller particles in the compacts which were caused by the impact effect of balls on particles shows more unfavorable surfaces and defects. Owing to the pinning effect of these defects, the hysteresis loss of the samples prepared by the ball-milling method is higher than that of the samples prepared by the sol-gel method.

As for the eddy current loss factor $\left(K_{\mathrm{e}}\right)$, it is dominated by the electric resistivity, which could by elevated by increasing the intrinsic resistivity, defects, residual stress and porosity or by decreasing the particle size [24]. Coating an insulating $\mathrm{Al}_{2} \mathrm{O}_{3}$ layer on the phosphate iron powders directly increases the intrinsic resistivity, and the electric resistivity is positively correlated to the $\mathrm{Al}_{2} \mathrm{O}_{3}$ content. However, the increased number of the pores and grain boundaries and the reduced particle size which resulted from the ball-milling process account for the different $K_{\mathrm{e}}$ of various SMC samples. Therefore, compared to the sample prepared by the sol-gel method, the contribution of the eddy current loss to the total core loss for the sample prepared by the ball milling is smaller.

\section{Conclusions}

Novel hybrid phosphate-alumina iron-based soft magnetic composites (SMCs) were successfully fabricated by both the sol-gel method and the ball-milling method. The hybrid insulating layers effectively enhanced the frequency stability of the permeability and reduced the total core loss in the SMCs. The SMCs prepared by both methods retained above $85 \%$ of the permeability and showed only approximately $50 \%$ of the total core loss of the phosphated samples prepared under the same process conditions. The loss separation results show that the contribution of the $P_{\mathrm{h}}$ and the $P_{\mathrm{e}}$ to the total core loss is inverse. The hysteresis loss in the samples prepared by the ball-milling method is greater than that prepared by the sol-gel method. These results suggest that the hybrid phosphate-alumina coating is promising, and can be used to obtain high-performance SMCs for electromagnetic devices at high frequency.

Author Contributions: Data curation, Y.P., L.Q. and X.W.; Investigation, Y.P.; Methodology, Y.P.; Resources, J.P. and W.L.; Supervision, W.L.; Writing - original draft, Y.P.; Writing - review \& editing, W.L. All authors have read and agreed to the published version of the manuscript.

Funding: This research was funded by the National Natural Science Foundation of China (Grant No 51971162, U1933112, 51671146), the Program of Shanghai Technology Research Leader (18XD1423800, 19XD1432100), Shanghai Automobile Industry Science and Technology Development Fund (No.1831) and the Fundamental Research Funds for the Central Universities.

Conflicts of Interest: The authors declare no conflict of interest.

\section{References}

1. Périgo, E.A.; Weidenfeller, B.; Kollár, P.; Füzer, J. Past, present, and future of soft magnetic composites. Appl. Phys. Rev. 2018, 5, 031301. [CrossRef]

2. Zhang, Z.; Xu, W.; Guo, T.; Jiang, Y.; Yan, M. Effect of processing parameters on the magnetic properties and microstructures of molybdenum permalloy compacts made by powder metallurgy. J. Alloy. Compd. 2014, 594, 153-157. [CrossRef]

3. Wang, X.; Pan, F.; Xiang, Z.; Zeng, Q.; Pei, K.; Che, R.; Lu, W. Magnetic vortex core-shell $\mathrm{Fe}_{3} \mathrm{O}_{4} @ \mathrm{C}$ nanorings with enhanced microwave absorption performance. Carbon 2020, 157, 130-139. [CrossRef]

4. Shokrollahi, H.; Janghorban, K. Soft magnetic composite materials (SMCs). J. Mater. Process. Technol. 2007, 189, 1-12. [CrossRef]

5. Bayramlı, E.; Gölgelioğlu, Ö.; Ertan, H.B. Powder metal development for electrical motor applications. J. Mater. Process. Technol. 2005, 161, 83-88. [CrossRef] 
6. Pan, Y.; Peng, J.; Qian, L.; Xiang, Z.; Lu, W. Effects of compaction and heat treatment on the soft magnetic properties of iron-based soft magnetic composites. Mater. Res. Express 2020, 7, 016115. [CrossRef]

7. Wang, J.; Fan, X.a.; Wu, Z.; Li, G. Intergranular insulated $\mathrm{Fe} / \mathrm{SiO}_{2}$ soft magnetic composite for decreased core loss. Adv. Powder Technol. 2016, 27, 1189-1194. [CrossRef]

8. Wu, C.; Huang, M.; Luo, D.; Jiang, Y.; Yan, $\mathrm{M}$. $\mathrm{SiO}_{2}$ nanoparticles enhanced silicone resin as the matrix for Fe soft magnetic composites with improved magnetic, mechanical and thermal properties. J. Alloy. Compd. 2018, 741, 35-43. [CrossRef]

9. Luo, D.; Wu, C.; Yan, M. Incorporation of the $\mathrm{Fe}_{3} \mathrm{O}_{4}$ and $\mathrm{SiO}_{2}$ nanoparticles in epoxy-modified silicone resin as the coating for soft magnetic composites with enhanced performance. J. Magn. Magn. Mater. 2018, 452, 5-9. [CrossRef]

10. Kabátová, M.; Dudrová, E.; Bruncková, H. The effect of calcination on morphology of phosphate coating and microstructure of sintered iron phosphated powder. Surf. Interface Anal. 2013, 45, 1166-1173. [CrossRef]

11. Luo, Z.; Fan, X.a.; Hu, W.; Luo, F.; Li, G.; Li, Y.; Liu, X.; Wang, J. Controllable $\mathrm{SiO}_{2}$ insulating layer and magnetic properties for intergranular insulating Fe-6.5wt.\%Si/SiO $\mathrm{S}_{2}$ composites. Adv. Powder Technol. 2019, 30, 538-543. [CrossRef]

12. Lei, J.; Zheng, J.; Zheng, H.; Qiao, L.; Ying, Y.; Cai, W.; Li, W.; Yu, J.; Lin, M.; Che, S. Effects of heat treatment and lubricant on magnetic properties of iron-based soft magnetic composites with $\mathrm{Al} 2 \mathrm{O} 3$ insulating layer by one-pot synthesis method. J. Magn. Magn. Mater. 2019, 472, 7-13. [CrossRef]

13. Qian, L.; Peng, J.; Xiang, Z.; Pan, Y.; Lu, W. Effect of annealing on magnetic properties of $\mathrm{Fe}_{2} / \mathrm{Fe}_{3} \mathrm{O}_{4}$ soft magnetic composites prepared by in-situ oxidation and hydrogen reduction methods. J. Alloy. Compd. 2019, 778, 712-720. [CrossRef]

14. Zhou, M.M.; Han, Y.; Guan, W.W.; Han, S.J.; Meng, Q.S.; Xu, T.T.; Su, H.L.; Guo, X.; Zou, Z.Q.; Yang, F.Y.; et al. Magnetic properties and loss mechanism of Fe-6.5wt\%Si powder core insulated with magnetic Mn-Zn ferrite nanoparticles. J. Magn. Magn. Mater. 2019, 482, 148-154. [CrossRef]

15. Peng, Y.; Yi, Y.; Li, L.; Ai, H.; Wang, X.; Chen, L. Fe-based soft magnetic composites coated with NiZn ferrite prepared by a co-precipitation method. J. Magn. Magn. Mater. 2017, 428, 148-153. [CrossRef]

16. Wu, S.; Sun, A.; Lu, Z.; Cheng, C.; Gao, X. Magnetic properties of iron-based soft magnetic composites with $\mathrm{SiO}_{2}$ coating obtained by reverse microemulsion method. J. Magn. Magn. Mater. 2015, 381, 451-456. [CrossRef]

17. Taghvaei, A.H.; Shokrollahi, H.; Janghorban, K. Properties of iron-based soft magnetic composite with iron phosphate-silane insulation coating. J. Alloy. Compd. 2009, 481, 681-686. [CrossRef]

18. Huang, M.; Wu, C.; Jiang, Y.; Yan, M. Evolution of phosphate coatings during high-temperature annealing and its influence on the Fe and FeSiAl soft magnetic composites. J. Alloy. Compd. 2015, 644, 124-130. [CrossRef]

19. Pittini-Yamada, Y.; Périgo, E.A.; de Hazan, Y.; Nakahara, S. Permeability of hybrid soft magnetic composites. Acta Mater. 2011, 59, 4291-4302. [CrossRef]

20. Liu, Y.; Yi, Y.; Shao, W.; Shao, Y. Microstructure and magnetic properties of soft magnetic powder cores of amorphous and nanocrystalline alloys. J. Magn. Magn. Mater. 2013, 330, 119-133. [CrossRef]

21. Digne, M.; Sautet, P.; Raybaud, P.; Toulhoat, H.; Artacho, E. Structure and Stability of Aluminum Hydroxides: A Theoretical Study. J. Phys. Chem. B 2002, 106, 5155-5162. [CrossRef]

22. Lomayeva, S.F.; Syugaev, A.V.; Maratkanova, A.N.; Shakov, A.A.; Rozanov, K.N.; Petrov, D.A.; Stergiou, C.A. Structure and microwave properties of Fe powders prepared by surfactant-assisted ball milling in organic media. J. Alloy. Compd. 2017, 721, 18-27. [CrossRef]

23. Taghvaei, A.H.; Shokrollahi, H.; Janghorban, K.; Abiri, H. Eddy current and total power loss separation in the iron-phosphate-polyepoxy soft magnetic composites. Mater. Des. 2009, 30, 3989-3995. [CrossRef]

24. Shokrollahi, H.; Janghorban, K. Effect of warm compaction on the magnetic and electrical properties of Fe-based soft magnetic composites. J. Magn. Magn. Mater. 2007, 313, 182-186. [CrossRef]

(C) 2020 by the authors. Licensee MDPI, Basel, Switzerland. This article is an open access article distributed under the terms and conditions of the Creative Commons Attribution (CC BY) license (http://creativecommons.org/licenses/by/4.0/). 